\title{
LEVANTAMENTO FITOSSOCIOLÓGICO E CARÁTER SUCESSIONAL DE UM TRECHO DE FLORESTA SECUNDÁRIA CILIAR EM JUQUITIBA, SP, BRASIL ${ }^{1}$
}

\author{
Rodrigo Trassi Polisel ${ }^{2}$
}

\begin{abstract}
RESUMO - O objetivo deste trabalho foi levantar as estruturas horizontal e vertical da comunidade arbórea em regeneração de trecho de floresta secundária ciliar de aproximadamente trinta anos de idade e conhecer quais as espécies mais abundantes em cada estrato da floresta, para se conhecer o grau sucessional deste trecho florestal. Foram levantados $800 \mathrm{~m}^{2}$ de área, sub-divididos em dezesseis parcelas de 10 x $5 \mathrm{~m}$, no interior das quais todos os indivíduos com DAP $\geq 1 \mathrm{~cm}$ foram amostrados e identificados para as análises horizontal (DR, FR, DoR, IVC e IVI), vertical (PSR e RNT) e mista, a partir do índice de valor de importância ampliado (IVIa). Foram amostrados 689 indivíduos, pertencentes a 38 famílias, 74 gêneros e 109 espécies. A densidade total foi de 8.614 indivíduos/ha. O índice de diversidade de Shannon foi de 3,99 e o de eqüabilidade de Pielou, 0,85. Tibouchina pulchra, Psychotria suterella e Endlicheria paniculata se destacaram com elevados valores de IVIa. Guarea macrophylla, Gomidesia anacardiaefolia, Xylopia langsdorffiana e Endlicheria paniculata obtiveram elevados valores de RNT, indicando adequada regeneração natural no trecho. As espécies secundárias iniciais e umbrófilas apresentaram a maior importância ecológica nesse trecho de floresta com trinta anos de pousio, com os maiores valores de posição sociológica e índice de valor de importância. Além destas, as espécies secundárias tardias presentes em todos os estratos sugerem o grau sucessional intermediário para o trecho estudado.
\end{abstract}

Palavras-chave: Floresta Ombrófila Densa; Estrutura vertical; Sucessão secundária.

\section{PHYTOSOCIOLOGICAL SURVEY AND SUCESSIONAL CHARACTER IN A PLOT OF SECONDARY RIPARIAN FOREST AT JUQUITIBA, SP, BRAZIL}

\begin{abstract}
The objective of this study was to evaluate the horizontal and vertical structures of tree community in regeneration in a fragment of a secondary riparian forest at approximately 30 years of age and to identify the most abundant species in each fragment of the forest to determine the sucessional stage. An area of 800 $\mathrm{m}^{2}$ was subdivided into 16 samples of $10 \times 5 \mathrm{~m}$ and all individuals with $\mathrm{DBH} \geq 1 \mathrm{~cm}$ were sampled and identified for the following analyzes: horizontal parameters (DR, FR, DoR, IVC and IVI), vertical parameters (PSR and RNR) and mixed parameters, from of value of increased importance index (IVIa). The survey measured 689 individuals, belonging to 38 families, 74 genus and 109 species. The total density was 8,614 individuals/ ha. The index of Shannon's diversity was 3.99 and the index of Pielou's equability was 0.85. Tibouchina pulchra, Psychotria suterella and Endlicheria paniculata obtained high values of IVIa. Guarea macrophylla, Gomidesia anacardiaefolia, Xylopia langsdorffiana and Endlicheria paniculata achieved high values of RNT, indicating adequate natural regeneration in the plot. The initial secondary and umbrophylous species showed the highest ecological importance in this fragment of the forest, with the highest values of sociologic position and importance index. Furthermore, the presence of late secondary species in all layers suggest that the studied fragment is in intermediate succession degree.
\end{abstract}

Keywords: Dense Ombrophylous Forest; Vertical structure; Secondary succession.

\footnotetext{
${ }^{1}$ Recebido em 27.08.2009 aceito para publicação em 13.08.2013.

${ }^{2}$ Programa de Pós-Graduação em Biologia Vegetal na Universidade Estadual de Campinas, Instituto de Biologia, SP, Brasil. E-mail: <nest_usp@yahoo.com.br>.
} 


\section{INTRODUÇÃO}

As florestas secundárias representam a cobertura vegetal predominante no Domínio Mata Atlântica. Com base no último inventário florestal realizado por Kronka et al. (2005), o Estado de São Paulo apresenta atualmente apenas $13,94 \%$ de sua cobertura florestal original. Entretanto, a metade dessa área foi classificada como capoeira e mesmo a categoria Mata agrupa florestas em estádios sucessionais iniciais e intermediários e não como em estádio avançado de sucessão ou primitivo, fitofisionomia praticamente inexistente no Estado.

A partir dos estudos florísticos e fitossociológicos da comunidade arbórea realizados nas últimas três décadas, grandes avanços foram realizados no sentido de se conhecer a composição e estrutura do estrato arbustivo-arbóreo da Floresta Ombrófila Densa. Porém todo esse esforço não foi realizado de maneira equivalente aos estratos inferiores da floresta, principalmente referente às plântulas e indivíduos jovens de espécies arbóreas (NEGRELLE, 2006). Nesse sentido, apesar de se conhecer muito bem a composição das espécies lenhosas das formações secundárias, pouco se sabe sobre os padrões de regeneração e de abundância dessas espécies nos remanescentes florestais alterados (Cielo Filho; Santin, 2002).

O arranjo das espécies ao longo do gradiente vertical combinado com a distribuição e o tamanho de fustes regula o balanço de energia no interior de uma floresta. É notório que florestas bem estratificadas suportam maior biodiversidade, porque apresentam maior diferenciação de nichos (HUNTER JUNIOR, 1990). Portanto, a estratificação vertical da floresta pode influenciar a riqueza, a diversidade, o crescimento e a produção de biomassa, sendo um importante indicador ecológico (SOUZA et al., 2003).

O estudo da estrutura vertical de florestas secundárias contribui, além disso, para se avaliar a regeneração das espécies arbóreas, que decorre da interação de processos naturais de restabelecimento do ecossistema florestal. É, portanto, parte do ciclo de crescimento da floresta e refere-se às fases iniciais de seu estabelecimento e desenvolvimento (MARANGON et al., 2008), o que se insere na compreensão dos determinantes sucessionais da floresta.

Esse estudo se propõe a levantar as estruturas horizontal e vertical da comunidade arbórea em regeneração de trecho de floresta secundária ciliar de aproximadamente trinta anos de idade e conhecer quais as espécies mais abundantes em cada estrato da floresta, para se conhecer o grau sucessional do trecho estudado.

\section{MATERIAIS E MÉTODOS}

O estudo foi realizado no município de Juquitiba, sudoeste da região metropolitana de São Paulo (RMSP) (235' 40”'S e 47²'18”O). O clima é classificado como Cwa, verões úmidos e invernos amenos, de acordo com a escala de Köeppen (CEPAGRI, 2008). Segundo coletas realizadas com auxílio de pluviômetro na propriedade ao lado da área de estudo no período de 2004 a 2007, o índice pluviométrico anual atingiu o valor médio de 1743,01 mm. O mês mais chuvoso foi janeiro com valores entre $286 \mathrm{~mm}$ (jan/2006) até 457 mm (jan/2005). O mês mais seco foi agosto com índices pluviométricos entre $4 \mathrm{~mm}$ (ago/2004) a $48 \mathrm{~mm}$ (ago/ 2005). Não há déficit hídrico no solo. As temperaturas média, mínima média e máxima média do município são, respectivamente, $20,3{ }^{\circ} \mathrm{C}, 16,4^{\circ} \mathrm{C}$ e $23,6^{\circ} \mathrm{C}$. A temperatura mínima verificada foi de $9,5^{\circ} \mathrm{C}$ e a máxima, 29,1 ${ }^{\circ} \mathrm{C}$ (CEPAGRI, 2008). Aúltima geada na área ocorreu em julho de 1998.

No interior da área de estudo foi aberta uma trincheira e o solo classificado em Argissolo VermelhoAmarelo Álico (PRADO, 2005). A vegetação é classificada como Floresta Ombrófila Densa Montana, de acordo com o sistema proposto por Veloso et al. (1991).

Segundo informações disponibilizadas pelos moradores do entorno, quase toda a região sofreu intenso corte raso para a produção de carvão nas décadas de 1960 a 1980 . Especificamente no local estudado, a vegetação possui cerca de trinta anos de pousio. Aliada à atividade carvoeira, a região sofre com a ação contínua de palmiteiros, que agem indiscriminadamente no interior das propriedades particulares. A caça de animais silvestres por moradores da região é outro impacto digno de nota.

O local estudado representa um fragmento secundário de vegetação ciliar de topografia plana, um baixio que se formou devido ao formato meândrico dos ribeirões no local. A profundidade do lençol freático ao longo das parcelas varia de 60 a $90 \mathrm{~cm}$ de profundidade. No verão, o lençol freático pode chegar a $20 \mathrm{~cm}$ de distância da superfície e no inverno, 1,20 m. Na encosta adjacente, há uma floresta em estádio avançado de regeneração. 
Foram alocadas 16 parcelas de 10 x $5 \mathrm{~m}\left(800 \mathrm{~m}^{2}\right)$, ocupando praticamente toda a área plana de vegetação secundária ciliar que se objetivou levantar. No interior de cada parcela, todos os indivíduos arbustivo-arbóreos com diâmetro à altura do peito (DAP) maior ou igual a $1 \mathrm{~cm}$ foram identificados e seus respectivos valores de DAP e altura registrados. Optou-se por esse critério mínimo de inclusão para selecionar os indivíduos já estabelecidos na área.

O material botânico foi coletado e identificado em campo e, posteriormente, teve sua identificação confirmada com o auxílio de bibliografia especializada e comparação com o acervo do Herbário D. Bento Pickel (SPSF). As exsicatas do material-testemunha das espécies registradas neste estudo estão depositadas neste mesmo herbário. O sistema de classificação adotado foi o APG II (APG, 2003).

Os parâmetros fitossociológicos considerados para a análise da estrutura horizontal estão descritos em Müeller-Dombois e Ellenberg (1974) e abrangeram a densidade (DR), a dominância (DoR), a freqüência relativas (FR) e os índices de valor de cobertura (IVC) e de importância (IVI), obtidos com o auxílio do programa FITOPAC (SHEPHERD, 2005).

Para a análise da estrutura vertical da comunidade regenerante, os indivíduos foram agrupados em três classes de altura, definidas segundo os critérios propostos por Souza (1990) e utilizado por Mariscal Flores (1993):

Estrato Inferior: árvore com $\mathrm{h}_{\mathrm{j}}<(\mathrm{h}-\mathrm{S})$;

Estrato Médio: árvore com $(\mathrm{h}-\mathrm{S}) \leq \mathrm{h}_{\mathrm{j}}<(\mathrm{h}+\mathrm{S})$ e Estrato Superior: árvore com $h_{j} \geq(h+S)$.

Em que: $\mathrm{h}=$ média das alturas dos indivíduos amostrados; $S$ = desvio padrão das alturas totais do estrato $\left(h_{\mathrm{j}}\right)$; $\mathrm{h}_{\mathrm{j}}=$ altura total da j-ésima árvore individual do respectivo estrato.

Para avaliar a estrutura vertical, foram utilizados os parâmetros posição sociológica (PS) e regeneração natural (RN). A posição sociológica representa a composição florística dos diversos estratos e o papel de cada espécie em cada um deles (SOUZA, 2003).

Com a estratificação, as estimativas de Posição Sociológica Absoluta (PSAi) e Relativa (PSRi), por espécie, são obtidas pela solução das expressões (Finol, 1971): $V_{i j}=V F_{i j} \cdot n_{i j} ; V_{j}=N_{j} / N^{*} 100 ; P A_{i}=\Sigma V F_{j} \cdot n_{i j}$
e PSR $=P_{i j} / \Sigma P S A_{i} * 100$

Em que: $V F_{i j}=$ valor fitossociológico da i-ésima espécie no j-ésimo estrato; $V F j=$ valor fitossociológico simplificado do j-ésimo estrato; $n_{i j}=$ número de indivíduos de i-ésima espécie no j-ésimo estrato; $N_{j}=$ número de indivíduos no j-ésimo estrato; $N=$ número total de indivíduos de todas as espécies em todos os estratos; $P S A_{i}=$ posição sociológica absoluta da i-ésima espécie e $P S R_{i}=$ posição sociológica relativa (\%) da i-ésima espécie.

Com base nos parâmetros fitossociológicos das estruturas horizontal e vertical, foi obtido o índice de valor de importância ampliado (IVIa) de cada espécie. Esse IVIa reflete a heterogeneidade e a irregularidade entre os estratos da vegetação (PEREIRA-SILVA ET al., 2004).

A regeneração natural foi avaliada a partir da abundância e freqüência de cada espécie em cada estrato considerado, de acordo com o proposto por Finol (1971) e modificado por Volpato (1994). Para cada classe de altura foi definido um valor de regeneração natural (RNC), que somados resultaram no valor da taxa de regeneração natural total (RNT), segundo as expressões: $\mathrm{RNC}_{\mathrm{ij}}=\mathrm{DR}_{\mathrm{ij}}+\mathrm{FR}_{\mathrm{ij}} / 2 \mathrm{e} \mathrm{RNT}_{\mathrm{ij}}=\Sigma \mathrm{RNC}_{\mathrm{ij}} / 3$.

Em que: $\mathrm{RNC}_{\mathrm{ij}}=$ estimativa da regeneração natural da i-ésima espécie na j-ésima classe de altura; $\mathrm{DR}_{\mathrm{ij}}=$ densidade relativa da i-ésima espécie na j-ésima classe de altura; FRij = freqüência relativa da i-ésima espécie na i-ésima classe de altura;m que: $\mathrm{RNT}_{\mathrm{ij}}=$ estimativa da regeneração natural total da população amostrada da i-ésima espécie.

Foram calculados o PSR, o IVI e o IVIa das espécies que ocorreram em cada estrato para se ter uma noção da importância de cada faixa de altura para a estrutura vertical da vegetação.

A diversidade florística foi estimada pelo índice de diversidade de Shannon ( $\mathrm{H}^{\prime}$ ) e a eqüabilidade pelo índice de Pielou (J'), descritos em Brower e Zar (1984). Uma curva de rarefação foi organizada com base na riqueza de espécies pelo número de indivíduos da amostra para se avaliar a suficiência amostral dos dados, com o auxilio do programa ECOSIM (GOTELLI; ENTSMINGER, 2004).

A classificação sucessional das espécies desse trabalho foi feita com base no trabalho realizado na 
Reserva Florestal do Morro Grande (RFMG) por Bernacci et al. (2006), que apresenta ampla listagem florística dos fragmentos daquela região. Foram delimitadas quatro categorias sucessionais: a) Pioneiras (P): espécies heliófitas de ciclo de vida curto, com todas as fases do ciclo de vida desenvolvidas sob alta luminosidade; b) Secundárias iniciais (S): espécies com capacidade de estabelecimento em pequenas clareiras. A esse grupo, incluem-se as pioneiras longevas; c) Secundárias tardias (T): espécies com capacidade de estabelecimento no sub-bosque de florestas em estádios sucessionais intermediários e avançados. As suas plântulas são ciófitas e os indivíduos adultos formam o dossel de florestas em estádios avançados de regeneração. São típicas de florestas maduras; d) Umbrófilas (U): espécies de sub-bosque, que completam todo o seu ciclo de vida no interior da floresta.

\section{RESULTADOS}

Em $800 \mathrm{~m}^{2}$ foram amostrados 689 indivíduos, pertencentes a 38 famílias, 74 gêneros e 109 espécies (Tabela 1). A densidade total de indivíduos de espécies arbustivas/arbóreas com DAP $\geq 1 \mathrm{~cm}$ no trecho amostral foi de $8.614 \mathrm{ind} / \mathrm{ha}$. O índice de diversidade de Shannon foi de 3,99 e o de eqüabilidade de Pielou, 0,85 . Com base na curva de rarefação, infere-se que a suficiência amostral para a riqueza no local está próxima de ser atingida (Figura 1 ). Vale ressaltar que não foi possível a instalação de mais unidades amostrais na área, devido às limitações impostas pela rede de drenagem no local.

A partir das alturas totais, foram definidos três estratos (SOUZA, 1990): o estrato inferior ( $\mathrm{h} \leq 1,97$ $\mathrm{m})$, o estrato médio $(1,97<\mathrm{h} \leq 6,61 \mathrm{~m})$ e o estrato superior (h > 6,61 m). A maior riqueza e abundância foram verificadas para o estrato médio, seguido pelo superior, assim também para os parâmetros de estrutura vertical e horizontal (Tabela 2), quando observado o valor destes parâmetros tratados para as espécies de ocorrência para cada estrato.

Com base na categorização sucessional, seis espécies foram classificadas como pioneira, com destaque para Tibouchina pulchra, o manacá-da-serra, que obteve o maior IVIa da comunidade, devido seus valores elevados de dominância $(19,86)$, e seguida por Clethra scabra. O manacá-da-serra, entretanto, não obteve valores expressivos de posição sociológica e regeneração natural
(Figura 2A e 2B), por não ter sido amostrado nos estratos inferiores da floresta. Pelo contrário, sua população já se apresentou de forma senescente na área.

A maior riqueza por categoria sucessional foi verificada para as espécies secundárias iniciais (43 espécies). Destacaram, em IVIa, as espécies: Gomidesia anacardiaefolia, Alchornea triplinervia e Myrcia formosiana dentro do grupo. A primeira apresentou índice de regeneração natural total expressivo $(22,48)$ (Figura 2B), muito superior as duas outras, que se destacaram pelos seus valores de dominância e densidade, parâmetros da estrutura horizontal que influenciaram no cálculo do IVI.

Foram registradas 27 espécies secundárias tardias. Entre elas, Myrcia aff. freyressiana, Pterocarpus rhorii e Guatteria australis possuíram IVIa expressivos (Tabela 1). Dessas, apenas a terceira apresentou valor de regeneração natural total elevado (Figura 2A e 2B), devido sua presença no estrato inferior. Convém ressaltar o comportamento de Xylopia langsdorffiana, que apesar de apresentar poucos indivíduos no trecho estudado, obteve elevado valor de regeneração natural, já que o cálculo relativo do índice na classe inferior foi superestimado devido ao baixo número de indivíduos amostrados neste estrato (Tabela 1).

A espécie Ocotea catharinensis é citada como em perigo de extinção, de acordo com a listagem de espécies ameaçadas do Instituto Brasileiro do Meio Ambiente e dos Recursos Naturais Renováveis - IBAMA (BRASIL, 2008). Já Ocotea daphnifolia está na categoria em perigo na listagem de espécies ameaçadas do Estado de São Paulo. Quiina magellano-gomezii e Ocotea odorifera estão na categoria vulnerável também desta listagem (SÃO PAULO, 2004).

Foram registradas 25 espécies umbrófilas na área de estudo. Psychotria suterella, Endlicheria paniculata, Guarea macrophyla, Rudgea jasminoides, Cyathea delgadii, Guapira opposita e Mollinedia schottiana foram as que apresentaram os maiores valores de IVIa (Figura 2A), apesar de apenas E. paniculata e G. macrophyla terem se destacado na regeneração natural (Figura 2B). Chomelia catharinae é uma arvoreta típica do sub-bosque florestal de trechos secundários que apresentou elevado valor de regeneração natural total (RNT) (Figura 2B). Neste grupo, duas espécies estão ameaçadas de extinção, ambas na categoria vulnerável, segundo a listagem do Estado de São Paulo (SÃO PAULO, 
Tabela 1 - Espécies amostradas no levantamento fitossociológico ordenadas em ordem decrescente de importância realizado em trecho de floresta secundária ciliar em Juquitiba, São Paulo, Brasil. Sendo P: pioneira; S: secundária inicial; T: secundária tardia; U: umbrófila; Ni, Nm e Ns: número de indivíduos dos estratos inferior, médio e superior, respectivamente; DR: densidade relativa; DoR: dominância relativa; FR: frequência relativa; IVI: índice de valor de importância (soma 300); PSR: posição sociológica relativa, em porcentagem; RNCi, RNCm e RNCs: regeneração natural das classes inferior, media e superior, respectivamente; RNT: regeneração natural total (soma 300); e IVIa: índice de valor de importância ampliado (soma: 400).

Table 1 - Species sampled in the phytosociological survey sorted in decreasing order of importance performed in the fragment of secondary riparian forest in Juquitiba, São Paulo, Brazil, where P: pioneer; S: initial secondary; T: late secondary; $U$ : umbrophylous; Ni, Nm and Ns: number of individuals of lower, medium and higher layer, respectively; DR: relative density; DoR: relative dominance; FR: relative frequency; IVI: value of importance index (sum: 300); PSR: relative sociologic position, in percentage; RNCi, RNCm and RNCs: natural regeneration of lower, medium and higher class, respectively; RNT: total natural regeneration (sum = 300); and IVIa: value of increased importance index $($ sum $=400)$.

\begin{tabular}{|c|c|c|c|c|c|c|c|c|c|c|c|c|c|}
\hline \multirow{2}{*}{ Espécies (Grupo sucessional) } & \multicolumn{8}{|c|}{ Estrutura horizontal } & \multicolumn{5}{|c|}{ Estrutura vertical } \\
\hline & $\mathrm{Ni}$ & $\mathrm{Nm}$ & Ns & DR & DoR & FR & IVI & PSR & $\mathrm{RNCi}$ & $\mathrm{RNCm}$ & RNCs & RNT & I \\
\hline Tibouchina pulchra Cogn. (P) & 0 & 0 & 18 & 2,60 & 19,86 & 2,81 & 25,27 & 0,60 & 0,00 & 1,41 & 8,04 & 9,44 & 25,87 \\
\hline Psychotria suterella Müll. Arg. (U) & 0 & 51 & 0 & 7,38 & 2,14 & 3,75 & 13,27 & 8,62 & 0,00 & 6,36 & 0,00 & 6,36 & 21,89 \\
\hline Endlicheria paniculata (Spreng.) J.F.Macbr (U) & 1 & 45 & 4 & 7,24 & 2,51 & 3,51 & 13,26 & 7,75 & 5,88 & 5,71 & 4,73 & 16,32 & 21,01 \\
\hline Gomidesia anacardiaefolia (Gardner) O. Berg (S) & 2 & 33 & 0 & 5,07 & 1,85 & 3,28 & 10,19 & 5,59 & 11,76 & 4,54 & 5,88 & 22,19 & 15,78 \\
\hline Guarea macrophyla Vahl. (U) & 2 & 35 & 0 & 5,35 & 0,91 & 3,51 & 9,77 & 5,93 & 11,76 & 4,83 & 5,88 & 22,48 & 15,70 \\
\hline Rudgea jasminoides (Cham.) Müll. Arg. (U) & 0 & 31 & 0 & 4,49 & 0,98 & 3,28 & 8,74 & 5,24 & 0,00 & 4,36 & 0,00 & 4,36 & 13,98 \\
\hline Alchornea triplinervia (Spreng.) Müll. Arg. (S) & 0 & 2 & 13 & 2,17 & 8,29 & 2,34 & 12,80 & 0,77 & 0,00 & 1,35 & 5,80 & 7,15 & 13,57 \\
\hline Cyathea delgadii Sternb. (U) & 0 & 14 & 6 & 2,89 & 5,72 & 2,34 & 10,95 & 2,57 & 0,00 & 2,40 & 2,68 & 5,08 & 13,52 \\
\hline Myrcia formosiana DC. (S) & 0 & 15 & 8 & 3,33 & 4,01 & 2,81 & 10,15 & 2,80 & 0,00 & 2,72 & 3,57 & 6,29 & 12,95 \\
\hline Myrcia aff. freyressiana (O. Berg) Kiaersk. (T) & 0 & 18 & 2 & 2,89 & 1,89 & 2,34 & 7,12 & 3,11 & 0,00 & 2,75 & 0,89 & 3,65 & 10,23 \\
\hline Guapira opposita (Vell.) Reitz (U) & 0 & 19 & 1 & 2,89 & 1,55 & 2,34 & 6,78 & 3,25 & 0,00 & 2,84 & 0,45 & 3,29 & 10,03 \\
\hline Mollinedia schottiana (Spreng.) Perkins (U) & 0 & 21 & 0 & 2,60 & 0,22 & 2,81 & 5,64 & 3,89 & 0,00 & 3,43 & 0,00 & 3,43 & 9,53 \\
\hline Attalea dubia (Mart.) Burret (S) & 0 & 0 & 6 & 0,87 & 6,47 & 1,41 & 8,75 & 0,20 & 0,00 & 0,70 & 2,68 & 3,38 & 8,95 \\
\hline Pterocarpus rhorii Vahl (T) & 0 & 16 & 1 & 2,46 & 1,07 & 2,34 & 5,88 & 2,74 & 0,00 & 2,58 & 0,45 & 3,02 & 8,62 \\
\hline Casearia sylvestris Sw. (S) & 0 & 17 & 3 & 2,89 & 0,64 & 2,11 & 5,64 & 2,97 & 0,00 & 2,55 & 1,34 & 3,89 & 8,61 \\
\hline Guatteria australis A. St-Hil. (T) & 1 & 12 & 1 & 2,03 & 0,74 & 2,34 & 5,11 & 2,07 & 5,88 & 2,23 & 3,39 & 11,50 & 7,18 \\
\hline Platymiscium floribundum Vog. (S) & 0 & 5 & 3 & 1,16 & 3,03 & 1,64 & 5,83 & 0,95 & 0,00 & 1,26 & 1,34 & 2,60 & 6,78 \\
\hline Guapira nitida (Schmidt) Lundell (U) & 0 & 11 & 0 & 1,59 & 0,84 & 2,34 & 4,78 & 1,86 & 0,00 & 2,14 & 0,00 & 2,14 & 6,64 \\
\hline Matayba intermedia Radlk. (S) & 0 & 3 & 2 & 0,72 & 4,35 & 0,94 & 6,01 & 0,57 & 0,00 & 0,73 & 0,89 & 1,62 & 6,58 \\
\hline Gomidesia sp. (S) & 1 & 12 & 0 & 1,88 & 0,12 & 2,11 & 4,11 & 2,03 & 5,88 & 2,11 & 2,94 & 10,93 & 6,14 \\
\hline Calycorectes australis D. Legrand (T) & 0 & 5 & 2 & 1,01 & 2,36 & 1,64 & 5,01 & 0,91 & 0,00 & 1,26 & 0,89 & 2,15 & 5,92 \\
\hline Ocotea dispersa (Ness) Mez (S) & 1 & 9 & 1 & 1,59 & 0,21 & 2,11 & 3,91 & 1,56 & 5,88 & 1,84 & 3,39 & 11,11 & 5,47 \\
\hline Clethra scabra Pers. (P) & 0 & 6 & 3 & 1,30 & 1,04 & 1,64 & 3,98 & 1,11 & 0,00 & 1,35 & 1,34 & 2,69 & 5,09 \\
\hline Mollinedia ulleana Perkins (U) & 0 & 1 & 0 & 1,45 & 0,24 & 1,64 & 3,33 & 1,69 & 0,00 & 1,70 & 0,00 & 1,70 & 5,02 \\
\hline Rollinia sericea (R.E.Fr.) R.E.Fr. (S) & 0 & 9 & 1 & 1,45 & 0,80 & 1,17 & 3,42 & 1,55 & 0,00 & 1,38 & 0,45 & 1,82 & 4,97 \\
\hline Miconia cubatanensis Hoehne (S) & 0 & 9 & 0 & 1,30 & 0,90 & 1,17 & 3,37 & 1,52 & 0,00 & 1,38 & 0,00 & 1,38 & 4,89 \\
\hline Rapanea umbellata (Mart.) Mez (S) & 0 & 5 & 3 & 1,16 & 1,14 & 1,64 & 3,93 & 0,95 & 0,00 & 1,26 & 1,34 & 2,60 & 4,88 \\
\hline Maytenus robusta Reissek (S) & 0 & 6 & 1 & 1,01 & 1,10 & 1,64 & 3,75 & 1,05 & 0,00 & 1,35 & 0,45 & 1,79 & 4,80 \\
\hline Esenbeckia grandiflora Mart. (U) & 0 & 8 & 0 & 1,16 & 0,29 & 1,87 & 3,32 & 1,35 & 0,00 & 1,29 & 0,00 & 1,29 & 4,67 \\
\hline Cabralea canjerana (Vell.) Mart. (T) & 0 & 6 & 2 & 1,16 & 0,91 & 1,41 & 3,47 & 1,08 & 0,00 & 1,23 & 0,89 & 2,12 & 4,55 \\
\hline Weinmania paullinifolia Pohl (S) & 0 & 0 & 1 & 0,14 & 3,71 & 0,23 & 4,09 & 0,03 & 0,00 & 0,12 & 0,45 & 0,56 & 4,12 \\
\hline Myrcia tenuivenosa Kiaersk. (U) & 0 & 8 & 0 & 1,16 & 0,11 & 1,17 & 2,44 & 1,35 & 0,00 & 1,17 & 0,00 & 1,17 & 3,79 \\
\hline Copaifera trapezifolia Hayne (T) & 1 & 6 & 1 & 1,16 & 0,14 & 1,41 & 2,71 & 1,05 & 5,88 & 1,23 & 3,39 & 10,50 & 3,76 \\
\hline Allophylus petiolulatus Radlk. (U) & 0 & 8 & 0 & 1,16 & 0,22 & 0,94 & 2,32 & 1,35 & 0,00 & 1,64 & 0,00 & 1,64 & 3,67 \\
\hline Eugenia cerasiflora Miq. (T) & 0 & 6 & 0 & 0,87 & 0,23 & 1,17 & 2,27 & 1,01 & 0,00 & 1,11 & 0,00 & 1,11 & 3,28 \\
\hline Prunus myrtifolia (L.) Urb. (S) & 0 & 2 & 3 & 0,72 & 0,81 & 1,17 & 2,70 & 0,44 & 0,00 & 0,76 & 1,34 & 2,10 & 3,14 \\
\hline Vernonia diffusa Less (P) & 0 & 0 & 2 & 0,29 & 2,04 & 0,47 & 2,80 & 0,07 & 0,00 & 0,23 & 0,89 & 1,13 & 2,87 \\
\hline Ocotea catharinensis Mez (T) & 0 & 5 & 0 & 0,72 & 0,40 & 0,70 & 1,83 & 0,85 & 0,00 & 0,79 & 0,00 & 0,79 & 2,68 \\
\hline
\end{tabular}


Tabela 1 - Cont.

Table 1 - Cont.

\begin{tabular}{|c|c|c|c|c|c|c|c|c|c|c|c|c|c|}
\hline \multirow{2}{*}{ Espécies (Grupo sucessional) } & \multicolumn{8}{|c|}{ Estrutura horizontal } & \multicolumn{5}{|c|}{ Estrutura vertical } \\
\hline & $\mathrm{Ni}$ & $\mathrm{Nm}$ & Ns & $\mathrm{DR}$ & DoR & $\mathrm{FR}$ & IVI & PSR & RNCi & $\mathrm{RNCm}$ & RNCs & RNT & I \\
\hline Chomelia catharinae (L.B. Sm. \& Downs) Steyerm. (U) & 1 & 4 & 0 & 0,72 & 0,06 & 1,17 & 1,96 & 0,68 & 5,88 & 0,94 & 2,94 & 9,76 & 2,64 \\
\hline Solanum swartzianum Roem. \& Schult. (S) & 0 & 3 & 1 & 0,58 & 0,73 & 0,70 & 2,01 & 0,54 & 0,00 & 0,38 & 0,45 & 0,83 & 2,55 \\
\hline Inga sessilis (Vell.) Mart. (S) & 0 & 1 & 2 & 0,43 & 1,38 & 0,47 & 2,28 & 0,24 & 0,00 & 0,32 & 0,89 & 1,21 & 2,52 \\
\hline Nectandra oppositifolia Ness. (S) & 0 & 3 & 1 & 0,58 & 0,49 & 0,70 & 1,77 & 0,54 & 0,00 & 0,61 & 0,45 & 1,06 & 2,31 \\
\hline Chionantus filiformis (Vell.) P.S. Green (T) & 0 & 0 & 1 & 0,14 & 1,86 & 0,23 & 2,24 & 0,03 & 0,00 & 0,12 & 0,45 & 0,56 & 2,27 \\
\hline Psychotria vellosiana Benth. (U) & 0 & 3 & 0 & 0,43 & 0,61 & 0,70 & 1,75 & 0,51 & 0,00 & 0,50 & 0,00 & 0,50 & 2,26 \\
\hline Cordia sellowiana Cham. (S) & 0 & 1 & 2 & 0,43 & 0,94 & 0,47 & 1,84 & 0,24 & 0,00 & 0,32 & 0,89 & 1,21 & 2,08 \\
\hline Erythroxylum argentinum O.E.Schulz (U) & 0 & 4 & 0 & 0,58 & 0,04 & 0,70 & 1,33 & 0,68 & 0,00 & 0,70 & 0,00 & 0,70 & 2,01 \\
\hline Slonea monosperma Vell. (T) & 0 & 4 & 0 & 0,58 & 0,02 & 0,70 & 1,30 & 0,68 & 0,00 & 0,59 & 0,00 & 0,59 & 1,98 \\
\hline Campomanesia xanthocarpa O. Berg (T) & 1 & 3 & 0 & 0,58 & 0,18 & 0,70 & 1,46 & 0,51 & 5,88 & 0,61 & 2,94 & 9,44 & 1,97 \\
\hline Myrcia pubipetala Miq. (S) & 0 & 1 & 2 & 0,43 & 0,53 & 0,70 & 1,67 & 0,24 & 0,00 & 0,44 & 0,89 & 1,33 & 1,91 \\
\hline Rupretchia laxiflora Meisn. (S) & 0 & 0 & 1 & 0,14 & 1,48 & 0,23 & 1,86 & 0,03 & 0,00 & 0,12 & 0,45 & 0,56 & 1,89 \\
\hline Myrceugenia mircioides (Cambess.) O. Berg (U) & 0 & 3 & 0 & 0,43 & 0,25 & 0,70 & 1,38 & 0,51 & 0,00 & 0,61 & 0,00 & 0,61 & 1,89 \\
\hline Casearia decandra Jacq. (S) & 0 & 3 & 0 & 0,43 & 0,12 & 0,70 & 1,26 & 0,51 & 0,00 & 0,61 & 0,00 & 0,61 & 1,77 \\
\hline Ocotea glaziovii Mez (T) & 0 & 3 & 0 & 0,43 & 0,06 & 0,70 & 1,20 & 0,51 & 0,00 & 0,61 & 0,00 & 0,61 & 1,71 \\
\hline Ocotea brachybotrya (Meisn.) Mez (U) & 0 & 3 & 0 & 0,43 & 0,02 & 0,70 & 1,16 & 0,51 & 0,00 & 0,61 & 0,00 & 0,61 & 1,67 \\
\hline Eugenia stigmatosa DC. (T) & 0 & 3 & 1 & 0,29 & 0,55 & 0,23 & 1,07 & 0,54 & 0,00 & 0,61 & 0,45 & 1,06 & 1,61 \\
\hline Geonoma schottiana Mart. (U) & 0 & 2 & 0 & 0,29 & 0,48 & 0,47 & 1,24 & 0,34 & 0,00 & 0,41 & 0,00 & 0,41 & 1,58 \\
\hline Myrtaceae 2 (s/c) & 0 & 4 & 0 & 0,29 & 0,14 & 0,47 & 0,90 & 0,68 & 0,00 & 0,70 & 0,00 & 0,70 & 1,58 \\
\hline Xylosma glaberrima Sleumer (S) & 0 & 3 & 0 & 0,43 & 0,12 & 0,47 & 1,02 & 0,51 & 0,00 & 0,38 & 0,00 & 0,38 & 1,53 \\
\hline Lamanonia ternata Vell. (S) & 0 & 3 & 0 & 0,43 & 0,19 & 0,23 & 0,85 & 0,51 & 0,00 & 0,61 & 0,00 & 0,61 & 1,36 \\
\hline Heisteria silvianii Schwacke (T) & 0 & 1 & 1 & 0,29 & 0,27 & 0,47 & 1,03 & 0,20 & 0,00 & 0,20 & 0,45 & 0,65 & 1,23 \\
\hline Myrtaceae 3 (s/c) & 0 & 0 & 1 & 0,14 & 0,81 & 0,23 & 1,19 & 0,03 & 0,00 & 0,12 & 0,45 & 0,56 & 1,22 \\
\hline Tabebuia alba (Cham.) Sandwith (S) & 0 & 2 & 0 & 0,29 & 0,09 & 0,47 & 0,85 & 0,34 & 0,00 & 0,29 & 0,00 & 0,29 & 1,19 \\
\hline Citronela paniculata (Mart.) R.A. Howard (S) & 0 & 1 & 1 & 0,29 & 0,46 & 0,23 & 0,98 & 0,20 & 0,00 & 0,32 & 0,45 & 0,77 & 1,18 \\
\hline Meliosma sellowii Urb. (T) & 0 & 3 & 0 & 0,43 & 0,11 & 0,47 & 1,01 & 0,17 & 0,00 & 0,20 & 0,00 & 0,20 & 1,18 \\
\hline Protium wildgrenii Engl. (T) & 0 & 1 & 1 & 0,29 & 0,19 & 0,47 & 0,95 & 0,20 & 0,00 & 0,32 & 0,45 & 0,77 & 1,15 \\
\hline Coccoloba warmingii Meisn. (S) & 0 & 2 & 0 & 0,29 & 0,05 & 0,47 & 0,81 & 0,34 & 0,00 & 0,41 & 0,00 & 0,41 & 1,15 \\
\hline Maprounea guianensis Aubl. (S) & 0 & 2 & 0 & 0,29 & 0,05 & 0,47 & 0,81 & 0,34 & 0,00 & 0,41 & 0,00 & 0,41 & 1,15 \\
\hline Marlierea tomentosa Cambess. (T) & 0 & 1 & 1 & 0,29 & 0,18 & 0,47 & 0,93 & 0,20 & 0,00 & 0,32 & 0,45 & 0,77 & 1,13 \\
\hline Bactris setosa Mart. (U) & 0 & 2 & 0 & 0,29 & 0,03 & 0,47 & 0,78 & 0,34 & 0,00 & 0,41 & 0,00 & 0,41 & 1,12 \\
\hline Myrcia fallax (Rich.) DC. (S) & 0 & 2 & 0 & 0,29 & 0,01 & 0,47 & 0,77 & 0,34 & 0,00 & 0,29 & 0,00 & 0,29 & 1,11 \\
\hline Tetrorchidium rubrivenium Poepp. (S) & 1 & 0 & 1 & 0,29 & 0,26 & 0,47 & 1,02 & 0,04 & 5,88 & 0,23 & 3,39 & 9,50 & 1,06 \\
\hline Myrtaceae 4 (s/c) & 0 & 0 & 1 & 0,14 & 0,64 & 0,23 & 1,02 & 0,03 & 0,00 & 0,12 & 0,45 & 0,56 & 1,05 \\
\hline Myrceugenia kleinii D. Legrand \& Kausel (U) & 0 & 2 & 0 & 0,29 & 0,11 & 0,23 & 0,64 & 0,34 & 0,00 & 0,29 & 0,00 & 0,29 & 0,98 \\
\hline Geonoma brevispatha Barb. Rodr. (U) & 0 & 2 & 0 & 0,29 & 0,07 & 0,23 & 0,60 & 0,34 & 0,00 & 0,41 & 0,00 & 0,41 & 0,94 \\
\hline Myrtaceae 6 (s/c) & 0 & 2 & 0 & 0,29 & 0,01 & 0,23 & 0,53 & 0,34 & 0,00 & 0,41 & 0,00 & 0,41 & 0,87 \\
\hline Ficus luschnathiana (Miq.) Miq. (S) & 0 & 1 & 0 & 0,14 & 0,28 & 0,23 & 0,66 & 0,17 & 0,00 & 0,20 & 0,00 & 0,20 & 0,83 \\
\hline Xylopia langsdorffiana St.Hilaire \& Tulasne (T) & 2 & 0 & 0 & 0,29 & 0,01 & 0,47 & 0,77 & 0,01 & 11,76 & 0,23 & 5,88 & 17,88 & 0,78 \\
\hline Ilex paraguariensis A. St-Hil. (S) & 0 & 0 & 1 & 0,14 & 0,32 & 0,23 & 0,69 & 0,03 & 0,00 & 0,12 & 0,45 & 0,56 & 0,72 \\
\hline Ocotea teleiandra (Meins.) Mez (T) & 0 & 1 & 0 & 0,14 & 0,17 & 0,23 & 0,55 & 0,17 & 0,00 & 0,20 & 0,00 & 0,20 & 0,72 \\
\hline Myrtaceae 5 (s/c) & 0 & 1 & 0 & 0,14 & 0,15 & 0,23 & 0,53 & 0,17 & 0,00 & 0,20 & 0,00 & 0,20 & 0,70 \\
\hline Cedrela fissilis Vell. (T) & 0 & 1 & 0 & 0,14 & 0,14 & 0,23 & 0,52 & 0,17 & 0,00 & 0,20 & 0,00 & 0,20 & 0,69 \\
\hline Myrtaceae 1 (s/c) & 0 & 1 & 0 & 0,14 & 0,12 & 0,23 & 0,50 & 0,17 & 0,00 & 0,20 & 0,00 & 0,20 & 0,67 \\
\hline Euterpe edulis Mart. (U) & 0 & 1 & 0 & 0,14 & 0,09 & 0,23 & 0,47 & 0,17 & 0,00 & 0,20 & 0,00 & 0,20 & 0,64 \\
\hline Aegiphila sellowiana Cham. (P) & 0 & 1 & 0 & 0,14 & 0,08 & 0,23 & 0,46 & 0,17 & 0,00 & 0,32 & 0,00 & 0,32 & 0,63 \\
\hline Rapanea ferruginea (Ruiz \& Pav.) Mez (P) & 0 & 0 & 1 & 0,14 & 0,20 & 0,23 & 0,58 & 0,03 & 0,00 & 0,12 & 0,45 & 0,56 & 0,61 \\
\hline Casearia obliqua Spreng. (S) & 0 & 1 & 0 & 0,14 & 0,07 & 0,23 & 0,44 & 0,17 & 0,00 & 0,20 & 0,00 & 0,20 & 0,61 \\
\hline Sapium glandulatum (Vell.) Pax (S) & 0 & 1 & 0 & 0,14 & 0,07 & 0,23 & 0,44 & 0,17 & 0,00 & 0,20 & 0,00 & 0,20 & 0,61 \\
\hline Schefflera angustissima (Marchal) Frodin (S) & 0 & 1 & 0 & 0,14 & 0,05 & 0,23 & 0,43 & 0,17 & 0,00 & 0,20 & 0,00 & 0,20 & 0,60 \\
\hline
\end{tabular}


Tabela 1 - Cont.

Table 1 - Cont

\begin{tabular}{|c|c|c|c|c|c|c|c|c|c|c|c|c|c|}
\hline \multirow{2}{*}{ Espécies (Grupo sucessional) } & \multicolumn{8}{|c|}{ Estrutura horizontal } & \multicolumn{5}{|c|}{ Estrutura vertical } \\
\hline & $\mathrm{Ni}$ & $\mathrm{Nh}$ & Ns & $\mathrm{DR}$ & DoR & FR & IVI & PSR & $\mathrm{RNCi}$ & $\mathrm{RNCm}$ & RNCs & RNT & I \\
\hline Mollinedia oligantha Perkins (U) & 0 & 1 & 0 & 0,14 & 0,04 & 0,23 & 0,42 & 0,17 & 0,00 & 0,20 & 0,00 & 0,20 & 0,59 \\
\hline Ocotea daphnifolia (Meisn.) Mez (T) & 0 & 1 & 0 & 0,14 & 0,04 & 0,23 & 0,42 & 0,17 & 0,00 & 0,20 & 0,00 & 0,20 & 0,59 \\
\hline Tachigali denudata (Lewis) Vell. (S) & 0 & 1 & 0 & 0,14 & 0,04 & 0,23 & 0,42 & 0,17 & 0,00 & 0,20 & 0,00 & 0,20 & 0,59 \\
\hline Eugenia oblongata O. Berg (T) & 0 & 0 & 1 & 0,14 & 0,16 & 0,23 & 0,54 & 0,03 & 0,00 & 0,12 & 0,45 & 0,56 & 0,57 \\
\hline Ocotea sp. (s/c) & 0 & 0 & 1 & 0,14 & 0,16 & 0,23 & 0,54 & 0,03 & 0,00 & 0,12 & 0,45 & 0,56 & 0,57 \\
\hline Ocotea odorifera (Vellozo) Rohwer (T) & 0 & 1 & 0 & 0,14 & 0,02 & 0,23 & 0,40 & 0,17 & 0,00 & 0,20 & 0,00 & 0,20 & 0,57 \\
\hline Persea wildenovii Kosterm. (T) & 0 & 1 & 0 & 0,14 & 0,02 & 0,23 & 0,40 & 0,17 & 0,00 & 0,20 & 0,00 & 0,20 & 0,57 \\
\hline Miconia inconspicua Miq. (P) & 0 & 1 & 0 & 0,14 & 0,01 & 0,23 & 0,39 & 0,17 & 0,00 & 0,20 & 0,00 & 0,20 & 0,56 \\
\hline Psidium cattleyanum Sabine (S) & 0 & 1 & 0 & 0,14 & 0,01 & 0,23 & 0,39 & 0,17 & 0,00 & 0,20 & 0,00 & 0,20 & 0,56 \\
\hline Quiina magellano-gomezii Schwacke (T) & 0 & 1 & 0 & 0,14 & 0,01 & 0,23 & 0,39 & 0,17 & 0,00 & 0,20 & 0,00 & 0,20 & 0,56 \\
\hline Rapanea gardneriana (A.DC.) Mez (S) & 0 & 1 & 0 & 0,14 & 0,01 & 0,23 & 0,39 & 0,17 & 0,00 & 0,20 & 0,00 & 0,20 & 0,56 \\
\hline Amaioua intermedia Mart. (S) & 0 & 1 & 0 & 0,14 & 0,00 & 0,23 & 0,38 & 0,17 & 0,00 & 0,20 & 0,00 & 0,20 & 0,55 \\
\hline Inga sp. (s/c) & 0 & 1 & 0 & 0,14 & 0,00 & 0,23 & 0,38 & 0,17 & 0,00 & 0,20 & 0,00 & 0,20 & 0,55 \\
\hline Micropholis crassipedicellata (Mart. \& Eichler) Pierre (T) & 0 & 1 & 0 & 0,14 & 0,00 & 0,23 & 0,38 & 0,17 & 0,00 & 0,20 & 0,00 & 0,20 & 0,55 \\
\hline Xylopia brasiliensis Spreng. (U) & 0 & 1 & 0 & 0,14 & 0,00 & 0,23 & 0,38 & 0,17 & 0,00 & 0,20 & 0,00 & 0,20 & 0,55 \\
\hline Zanthoxylum rhoifolium Lam. (S) & 0 & 1 & 0 & 0,14 & 0,01 & 0,23 & 0,38 & 0,17 & 0,00 & 0,20 & 0,00 & 0,20 & 0,55 \\
\hline Sorocea bonplandii (Baill.) W.C.Burger (U) & 0 & 0 & 1 & 0,14 & 0,13 & 0,23 & 0,51 & 0,03 & 0,00 & 0,12 & 0,45 & 0,56 & 0,54 \\
\hline Eugenia florida DC. (S) & 0 & 0 & 1 & 0,14 & 0,12 & 0,23 & 0,50 & 0,03 & 0,00 & 0,12 & 0,45 & 0,56 & 0,53 \\
\hline Bathysa australis (A. St.-Hil.) Benth. \& Hook. F. (S) & 1 & 0 & 0 & 0,14 & 0,00 & 0,23 & 0,38 & 0,01 & 5,88 & 0,12 & 2,94 & 8,94 & 0,39 \\
\hline Gomidesia spectabilis (DC) O. Berg. (S) & 1 & 0 & 0 & 0,14 & 0,00 & 0,23 & 0,38 & 0,01 & 5,88 & 0,12 & 2,94 & 8,94 & 0,39 \\
\hline Pouteria bullata (S. Moore) Baehni (T) & 1 & 0 & 0 & 0,14 & 0,00 & 0,23 & 0,38 & 0,01 & 5,88 & 0,12 & 2,94 & 8,94 & 0,39 \\
\hline TOTAL & 17 & 560 & 112 & 100 & 100 & 100 & 300 & 100 & 100 & 100 & 100 & 300 & 400 \\
\hline
\end{tabular}

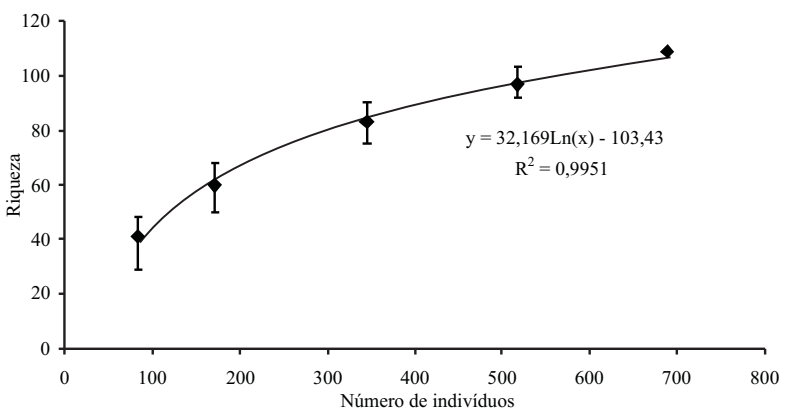

Figura 1 - Curva de rarefação da riqueza com o coeficiente de correlação de Sperman baseada no número de indivíduos na amostra em trecho de floresta secundária ciliar em Juquitiba, SP, Brasil.

Figure 1 -Richness rarefaction curve with the coeficient of Sperman's correlaction based on the number of individuals in the sample in the fragment of the secondary riparian fores in Juquitiba, SP, Brazil.

2004): Euterpe edulis e Myrceugenia kleinii.

\section{DISCUSSÃO}

Apesar do relativo conhecimento da flora e estrutura da comunidade arbórea em Floresta Ombrófila
Tabela 2 - Parâmetros fitossociológicos das estruturas horizontal e vertical de trecho de floresta secundária ciliar em Juquitiba, SP, Brasil, em que: S: riqueza; N: número de indivíduos; PSR: posição sociológica relativa; IVI: índice de valor de importância; IVIa: índice de valor de importância ampliado; e D: densidade absoluta (ind/ha).

Table 2 - Phytosociologicals parameters of the horizontal and vertical structures of the fragment of secondary riparian forest in Juquitiba, SP, Brazil, where: S: richness; $N$ : number of individuals; PSR: relative sociologic position; IVI: value of importance index; IVIa: value of increased importance index; and D: absolute density (ind/ha).

\begin{tabular}{lcccccc}
\hline Parâmetros & $\mathrm{S}$ & $\mathrm{N}$ & $\mathrm{PSR}$ & $\mathrm{IVI}$ & IVIa & $\mathrm{D}$ \\
\hline Inferior & 14 & 17 & 27,2 & 55,4 & 82,6 & 213 \\
Médio & 90 & 560 & 98,7 & 246,4 & 345,1 & 7000 \\
Superior & 45 & 112 & 43,6 & 188,5 & 232,2 & 1400 \\
TOTAL & 109 & 689 & 100 & 300 & 400 & 8613 \\
\hline
\end{tabular}

Densa nos Estados do Sul e Sudeste brasileiro, estudos com enfoque na distribuição vertical das espécies arbóreas ainda são incipientes, devido à dificuldade amostral e identificação das espécies em estágios juvenis (PRATA, 2009; NEGRELLE, 2006). Desta forma, índices como a posição sociológica (PS), a 
regeneração natural ( $R N$ ) e o índice de valor de importância ampliado (IVIa) são pouco utilizados, o que dificulta a comparação dos resultados encontrados neste trecho estudado com outros trabalhos.

Pela análise da estrutura vertical, é possível ter uma idéia da importância da espécie ou de guildas sucessionais para um trecho florestal, considerando
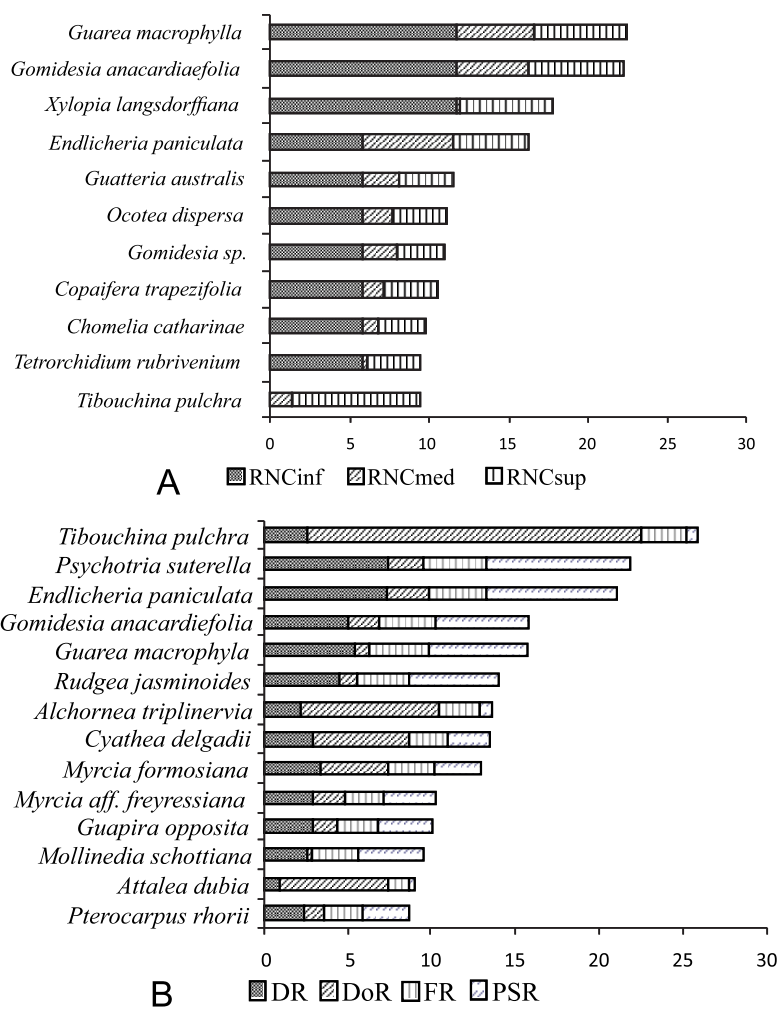

Figura 2 - (A) Regeneração Natural Total (RNT) e (B) Índice de Valor de Importância Ampliado (IVIa) das espécies que somam a metade desses parâmetros em trecho de floresta secundária ciliar em Juquitiba, SP, Brasil, em que: RNCi, RNCm e RNCs: regeneração natural das classes inferior, media e superior, respectivamente; DR: densidade relativa; DoR dominância relativa; FR: frequência relativa; e PSR: posição sociológica relativa.

Figure 2 - (A) Total natural regeneration (RNT) and (B) Value of increased importance index (IVIa) for the species that sum the half of these parameters in a fragment of secondary riparian forest in Juquitiba, SP, Brazil, where: $R N C i, R N C m$ and $R N C s$ : natural regeneration of lower, medium and higher class, respectively; $D R$ : relative density; DoR: relative dominance; FR: relative frequency; and PSR: relative sociologic position. a sua participação nos estratos verticais. Aquelas espécies que possuírem um maior número de indivíduos representantes em cada um desses estratos certamente apresentarão uma maior importância ecológica no povoamento em estudo (MARANGON et al., 2008).

Nessa perspectiva, as espécies secundárias iniciais e umbrófilas apresentaram a maior importância ecológica no trecho de floresta estudado, pois a primeira categoria revelou possuir a maior riqueza em todos os estratos e a maior abundância nos estratos inferior e superior da floresta. Já a categoria das espécies umbrófilas apresentou parâmetros expressivos de IVI e PSR nos estratos inferior e médio (Figura 3).

As espécies pioneiras foram bem representadas por Tibouchina pulchra e Clethra scabra nos estratos superiores. Segundo Tabarelli et al. (1994), essas duas espécies estão associadas à sucessão secundária de áreas que sofreram impacto antrópico severo, como é o caso do trecho de mata estudado, que foi utilizado para a produção de carvão vegetal.

Além disso, não se pode desconsiderar o grupo das espécies secundárias tardias, que ocorreram em todos os estratos, o que se depreende a evolução do quadro sucessional do fragmento ao longo do tempo.

Nessa categoria sucessional, certas espécies são típicas de florestas em estádio avançado de regeneração ou florestas que não sofreram corte raso e sua presença nesse trecho de floresta secundária ciliar nos estratos inferiores indica que o processo de sucessão secundária está por reintroduzi-las na área novamente. Tais espécies são citadas por Catharino et al. (2006) que também trabalharam no Planalto Paulistano na região de Cotia, SP, e estão listadas abaixo, organizadas em ordem decrescente de IVIa no trecho estudado: Copaifera trapezifolia $(3,76)$, Ocotea catharinensis $(2,68)$, Eugenia stigmatosa $(1,61)$, Ocotea daphnifolia $(0,59)$, Quiina magellano-gomezii $(0,56)$, Micropholis crassipedicellata $(0,55)$ e Pouteria bullata $(0,39)$.

Ogata e Gomes (2006) realizaram levantamento com todos os indivíduos lenhosos com altura maior que $1 \mathrm{~m}$ em fragmento de Floresta Ombrófila Densa em Cotia, SP. Os autores encontraram 79 espécies arbóreas na área que possui em torno de cinqüenta anos de pousio e ação antrópica moderada (parque municipal), a qual dificulta a regeneração natural das espécies nativas. As espécies com maior abundância neste trabalho foram Croton floribundus, Psychotria longipes e Cupania 


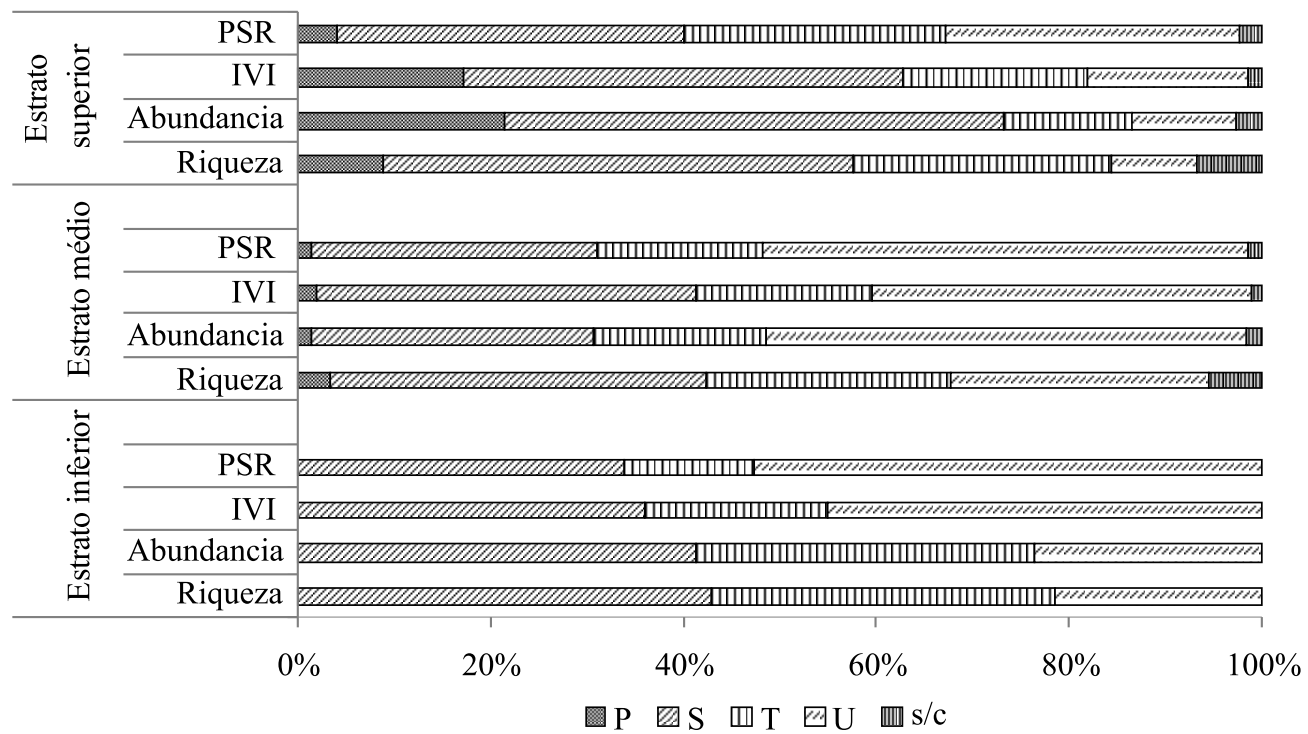

Figura 3 - Síntese dos parâmetros fitossociológicos das estruturas horizontal e vertical por grupo sucessional e por estrato de um trecho de floresta secundária ciliar em Juquitiba, SP, Brasil, em que: P: pioneira; S: secundária inicial; T: secundária tardia; e U: umbrófila.

Figure 3 - Summary of phytosociologicals parameters of horizontal and vertical structures for sucessional group and for the layer in the fragment of secondary riparian forest in Juquitiba, SP, Brazil, where: P: pioneer; S: initial secondary; T: late secondary; and U: umbrophylous.

vernalis, ao contrário do presente estudo, que apresentou as espécies Tibouchina pulchra e Clethra scabra, como as mais abundantes no local. A área de estudo em Juquitiba encontra-se na Floresta Ombrófila Densa, enquanto que o estudo de Ogata e Gomes (2006) foi realizado na transição entre esta fisionomia e a Floresta Estacional Semidecidual.

Em relação ao levantamento realizado em Itapoá, SC, por Negrelle (2006), em fragmento preservado de Floresta Ombrófila Densa de terras baixas, foram levantados todos os indivíduos arbóreos também com altura maior que $1 \mathrm{~m}$. Nesse trabalho foram registradas 135 espécies, valor correspondente ao grau avançado de regeneração da área.

Por todas essas características, é possível afirmar que o trecho estudado em Juquitiba, possui elevada riqueza (109 espécies) e diversidade $(3,99)$ de espécies arbóreas, além de efetivo número de espécies secundárias tardias em processo de regeneração.

Ao longo da estrutura vertical do trecho estudado, há uma substituição de guildas sucessionais, com o predomínio de espécies secundárias nos estratos superior e médio e espécies secundárias tardias e umbrófilas no estrato inferior. Assim, é possível classificar a vegetação estudada em estádio médio de regeneração.

\section{AGRADECIMENTOS}

O autor agradece ao proprietário Sr. Wilson e ao caseiro João Barreto (in memorian) pela autorização em realizar esse estudo no interior da propriedade e o auxílio nas atividades de campo. Os seguintes taxonomistas contribuíram para a identificação e confirmação das espécies registradas: Profa. Dra. Ariane Peixoto (Monimiaceae), Msc. Geraldo Franco (várias famílias), Dr. João Batista Baitello (Lauraceae), Msc Osny Tadeu Aguiar (Myrtaceae), Prof. Dr. Renato Goldenberg (Melastomataceae) e Msc. Rubens Luiz Coelho (Sapindaceae). O autor agradece ao Prof. Dr. Athylla Miklos da Faculdade de Filosofia, Letras e Ciências Humanas da Universidade de São Paulo pelo auxílio na classificação do solo na área de estudo.

\section{REFERÊNCIAS}

ANGIOSPERM PHYLOGENY GROUP II. - APG II. An update of the Angiosperm Phylogeny Group

Revista Árvore, Viçosa-MG, v.37, n.5, p.789-799, 2013 
classification for the orders and families of flowering plants: APG II. Botanical Journal of the Linnaeau Society, v.141, n.4, p.399436, 2003.

BERNACCI, L. C. et al. O efeito da fragmentação florestal na composição e riqueza de árvores na região da Reserva do Morro Grande (Planalto de Ibiúna, SP). Revista do Instituto

Florestal, v.18, p.121-166, 2006.

BRASIL. Instrução Normativa n. 6, de 23 de setembro de 2008. Dispõe sobre a Lista das espécies da flora brasileira ameaçada de extinção.

BROWER, J. E. \& ZAR, J. H. Field and laboratory methods for general ecology. Boston: Brown Publishers, 1984. 180p.

CATHARINO, E. L. M. et al. Aspectos da composição e diversidade do componente arbóreo das florestas da Reserva Florestal do Morro Grande, Cotia, SP. Biotaneotropica, v.6, n.2, 2006.<http://www.biotaneotropica.org.br/ v6n2/pt/abstract?article+bn00306022006> Último acesso em: 10 de maio de 2009.

\section{CEPAGRI. Centro de Pesquisa}

Metereológica e Climática Aplicada à Agricultura. 2008. <http://

www.cpa.unicamp.br/outras_informacoes _clima_muni_299.html> Ultimo acesso em: 05 de maio de 2009.

CIELO FILHO, R.; SANTIN, D. A. Estudo florístico e fitossociológico de um fragmento florestal urbano - Bosque dos Alemães, Campinas, SP. Revista Brasileira de Botânica., v.25, n.3, p.291-301, 2002.

FINOL, U. H. Nuevos parametros a considerarse en el analisis estrutural de las selvas virgenes tropicales. Revista Forestal Venezolana, v.18, n.12, p.29-42, 1971.

HUNTER JUNIOR, M. L. Wildlife forests, and forestry: principles of managing forests for biological diversity. New Jersey: PrenticeHall, 1990. 370p.

GOTELLI, N. J. \& ENTSMINGER, G. L. EcoSim:
Null models software for ecology. Version 7. Acquired Intelligence Inc. \& Kesey-Bear. Jericho, VT 05465. 2004. <http:// garyentsminger.com/ecosim/index.htm> Último acesso em: 15 de maio de 2009 .

KRONKA, F. J. N. et al. Inventário florestal da vegetação natural do Estado de São Paulo. São Paulo: Imprensa Oficial do Estado de São Paulo, Secretaria do Meio Ambiente, Instituto Florestal, 2005. 190p.

MARANGON, L. C. et al. Regeneração natural em um fragmento de Floresta Estacional Semidecidual em Viçosa, Minas Gerais. Revista Árvore, v.32, n.1, p.183-191, 2008.

MARISCAL FLores, E. J. Potencial produtivo e alternativas de manejo sustentável de um fragmento de Mata Atlântica secundária, município de Viçosa, Minas Gerais. 2003. 165f. Dissertação (Mestrado em Ciência Florestal) Universidade Federal de Viçosa, Viçosa, MG, 2003.

MÜELLER-DOMBOIS, D.; ELLENBERG, H. Aims and methods of vegetation ecology. New York: John Wiley \& Sons, 1974. 365p.

NEGRELLE, R. R. B. Composição florística e estrutura vertical de um trecho de Floresta Ombrófila Densa de Planície Quaternária. Hoehnea, v.33, n.3, p.261-289, 2006.

OGATA, H. \& GOMES, E. P. C. Estrutura e composição da vegetação no Parque CEMUCAM, Cotia, SP. Hoehnea, v.33, n.3, p.371-384, 2006.

PEREIRA-SILVA, E. F. L. et al. Florística e fitossociologia dos estratos arbustivo e arbóreo de um remanescente de cerradão em uma Unidade de Conservação do Estado de São Paulo. Reviata Brasileira de Botânica, v.27, n.3, p.533-544, 2004.

PRADO, H. Solos do Brasil. Piracicaba: FUNEP, 2005.

PRATA, E. M. B. Estrutura e composição 
de espécies arbóreas em um trecho de Floresta Ombrófila Densa no litoral norte do Estado de São Paulo e padrões de similaridade florística em escala regional. 2009. 106f. Dissertação (Mestrado em Biologia Vegetal) - Universidade Estadual de São Paulo, Rio Claro, 2009.

SÃO PAULO. Resolução n. 48, de 22 de setembro de 2004. Dispõe sobre a Listagem de plantas ameaçadas de extinção no Estado de São Paulo.

SHEPHERD, G. J. Manual de usuário: Programa Fitopac. Campinas: Universidade Estadual de Campinas, 2005.
SOUZA, A. L. Manejo florestal. Viçosa, MG: Universidade Federal de Viçosa, 1990. (Notas de aula)

SOUZA, D. R. et al. Emprego de análise multivariada para estratificação vertical de florestas ineqüiâneas. Revista Árvore, v.27, n.1, p.59-63, 2003.

TABARELLI, T.; VILLANI, J.P.; MANTOVANI, W. Estudo comparativo da vegetação de dois trechos de floresta secundária no Núcleo Santa Virgínia, Parque Estadual da Serra do Mar, SP. Revista do Instituto Florestal., v.6, n.1, p.1-11, 1994.

VELOSO, H. P.; RANGEL FILHO, A. L. R.; LIMA, J. C. A. Classificação da vegetação brasileira adaptada a um sistema universal. Rio de Janeiro: Fundação IBGE, 1991. 95p.

VOLPATO, M. M. L. Regeneração natural em uma floresta secundária no domínio de Mata Atlântica: uma análise fitossociológica. 1994. 123f. Dissertação (Mestrado em Ciência Florestal) Universidade Federal de Viçosa, Viçosa, MG, 1994. 
